\title{
EXPANSION OF DISCRETE AND CLOSURE-PRESERVING FAMILIES
}

\author{
TAKEMI MIZOKAMI
}

(Communicated by Dennis Burke)

\begin{abstract}
In this paper, we define the classes of $d$-IP-expandable spaces and IP-expandable spaces, and study their properties and relations with orthocompact spaces and nonarchimedean quasi-metrizable spaces.
\end{abstract}

1. Introduction. Following [1], a space $X$ is called CP-expandable if for each closure-preserving family $\mathcal{F}=\left\{F_{\lambda}: \lambda \in \Lambda\right.$ \} of closed subsets of $X$ and for each family $\mathcal{U}=\left\{U_{\lambda}: \lambda \in \Lambda\right\}$ of open subsets of $X$ such that $F_{\lambda} \subset U_{\lambda}$ for each $\lambda$, there exists a closure-preserving family $\mathcal{V}=\left\{V_{\lambda}: \lambda \in \Lambda\right\}$ of open subsets of $X$ such that $F_{\lambda} \subset V_{\lambda} \subset \bar{V}_{\lambda} \subset U_{\lambda}$ for each $\lambda$. In this paper, we introduce the classes of IP-expandable spaces and $d$-IP-expandable spaces by replacing "closure-preserving" with other conditions. Our main purpose is to study the properties of these classes and the relations with orthocompact spaces and nonarchimedean quasi-metrizable spaces.

All spaces are assumed to be $T_{1}$ topological spaces and $\mathrm{N}$ always denotes the set of natural numbers.

2. D-IP-expandability and IP-expandability. We state the definitions of $d$-IP-expandability and IP-expandability.

DEFINITION 2.1. We call a space $X d$-IP-expandable if for a discrete family $\mathcal{F}=\left\{F_{\lambda}: \lambda \in \Lambda\right\}$ of closed subsets of $X$ and a family $\mathcal{U}=\left\{U_{\lambda}: \lambda \in \Lambda\right\}$ of open subsets of $X$ such that $F_{\lambda} \subset U_{\lambda}$ for each $\lambda$, there exists an interior-preserving family $\mathcal{V}=\left\{V_{\lambda}: \lambda \in \Lambda\right\}$ of open subsets of $X$ such that $F_{\lambda} \subset V_{\lambda} \subset U_{\lambda}$ for each $\lambda$.

DEFINITION 2.2. We call a space $X$ IP-expandable if for a closure-preserving family $\mathcal{F}=\left\{F_{\lambda}: \lambda \in \Lambda\right\}$ of closed subsets of $X$ and a family $\mathcal{U}=\left\{U_{\lambda}: \lambda \in \Lambda\right\}$ of open subsets of $X$ such that $F_{\lambda} \subset U_{\lambda}$ for each $\lambda$, there exists a family $\mathcal{V}=\left\{V_{\lambda}: \lambda \in \Lambda\right\}$ of open subsets of $X$ such that $F_{\lambda} \subset V_{\lambda} \subset U_{\lambda}$ for each $\lambda$ and $\left\{V_{\lambda}: \lambda \in \Lambda\right\}$ is interior-preserving in $X$.

In either case, we call $\mathcal{V}$ the IP-expansion of $₹$ with respect to $\mathcal{U}$ in $X$. A space $X$ is called $(\sigma-)$ orthocompact if every open cover of $X$ has a $(\sigma$-)interior-preserving open refinement.

Proposition 2.3. If a space $X$ is collectionwise normal, then $X$ is $d$-IPexpandable.

PROPOSITION 2.4. If a space $X$ is orthocompact, then $X$ is d-IP-expandable.

Received by the editors December 26, 1985 and, in revised form, August 25, 1986.

1980 Mathematics Subject Classification (1985 Revision). Primary 54D20.

Key words and phrases. Interior-preserving, orthocompact, nonarchimedean quasi-metrizable, $d$-IP-expandable. 
ProOF. Let $\mathcal{F}=\left\{F_{\lambda}: \lambda \in \Lambda\right\}$ and $U=\left\{U_{\lambda}: \lambda \in \Lambda\right\}$ be the same pair of families as in Definition 2.1. Assume $F_{\lambda} \cap U_{\lambda^{\prime}}=\varnothing$ if $\lambda \neq \lambda^{\prime}$. Since $X$ is orthocompact, there exists an interior-preserving open refinement $\mathcal{W}$ of an open cover $U \cup\{X-\bigcup \mathcal{F}\}$. Setting $V_{\lambda}=S\left(F_{\lambda}, \mathcal{W}\right)$ for each $\lambda$, we have the IP-expansion $\mathcal{V}=\left\{V_{\lambda}: \lambda \in \Lambda\right\}$ of $\mathcal{F}$ with respect to $U$.

In [5], Michael constructed a normal, noncollectionwise normal, metacompact space $X$. By Proposition 2.4, $X$ is $d$-IP-expandable. Thus, the converse of Proposition 2.3 is not true. Also, Scott constructed a countably compact space $X$ which is not orthocompact [8, Example 4.5]. Obviously, $X$ is $d$-IP-expandable. Hence the converse of Proposition 2.4 is also not true. The following gives a simple sufficient condition for a $d$-IP-expandable space to be orthocompact.

THEOREM 2.5. If a space $X$ is submetacompact and d-IP-expandable, then $X$ is orthocompact.

ProOF. Let $\mathcal{U}$ be an open cover of $X$. Since $X$ is submetacompact, that is $\theta$ refinable, by [11] there exists an open refinement $\bigcup_{n=1}^{\infty} \mathcal{U}_{n}$ of $\mathcal{U}$ and a closed cover $\left\{F_{n}: n \in \mathbf{N}\right\}$ of $X$ such that for each $n, U_{n}$ covers $F_{n}$ and $U_{n}$ is point-finite at each point of $F_{n}$. For each $n, k \in \mathbf{N}$, set the closed set by

$$
E_{n k}=\left\{x \in F_{n}: \operatorname{ord}\left(x, \mathcal{U}_{n}\right) \leq k\right\},
$$

where $\operatorname{ord}\left(x, \mathcal{U}_{n}\right)=\left|\left\{U \in \mathcal{U}_{n}: x \in U\right\}\right|$. Then $\left\{E_{n k}: n, k \in \mathbf{N}\right\}$ satisfies the following conditions

(1) For each $n, k, \bigcup_{k=1}^{\infty} E_{n k}=F_{n}$ and $E_{n k} \subset E_{n k+1}$.

(2) For each $n, E_{n 1}$ is the union of a discrete family $\mathcal{E}_{n 1}$ of closed subsets of $F_{n}$ such that each $E \in \mathcal{E}_{n 1}$ is contained in some $U(E) \in \mathcal{U}_{n}$.

(3) For each $n$ and each $k \geq 2$, if $T$ is a closed subset of $E_{n k}$ such that $T \cap E_{n k-1}=$ $\varnothing$, then $T$ is the union of a discrete family $\mathcal{E}(T)$ of closed subsets of $E_{n k}$ such that each $E \in \mathcal{E}(T)$ is contained in some $U(E) \in \mathcal{U}_{n}$.

Let $n \in \mathbf{N}$ be fixed for a while. Since $X$ is $d$-IP-expandable, there exists the IP-expansion $\mathcal{V}_{n 1}$ of $\mathcal{E}_{n 1}$ with respect to $\left\{U(E): E \in \mathcal{E}_{n 1}\right\}$. By (3) and by $d$-IPexpandability of $X$ again, there exists the IP-expansion $V_{n 2}$ of $\mathcal{E}\left(E_{n 2}-\bigcup \mathcal{V}_{n 1}\right)$ with respect to $\left\{U(E): E \in \mathcal{E}\left(E_{n 2}-\bigcup \mathcal{V}_{n 1}\right)\right.$. Repeating this process, we can get a sequence $\left\{\mathcal{V}_{n k}: k \in \mathbf{N}\right\}$ of IP-expansions. It is easy to see that $\bigcup\left\{\mathcal{V}_{n k}: n, k \in \mathbf{N}\right\}$ is a $\sigma$-interior-preserving open refinement of $\mathcal{U}$. By $[3], X$ is countably metacompact. Let $\left\{V_{n k}: n, k \in \mathbf{N}\right\}$ be a point-finite open refinement of $\left\{\bigcup \mathcal{V}_{n k}: n, k \in \mathbf{N}\right\}$ such that $V_{n k} \subset \bigcup \mathcal{V}_{n k}$ for each $n, k$. It is easy to see that

$$
\bigcup\left[\left\{V_{n k} \cap V: V \in \mathcal{V}_{n k}\right\}: n, k \in \mathbf{N}\right]
$$

is an interior-preserving open refinement of $\mathcal{U}$.

The converse of Theorem 2.5 is not true, because there is a noncountably metacompact orthocompact space [8, Example 4.2]. A quasi-metric $d$ on a set $X$ with the property that $d(x, z) \leq \max \{d(x, y), d(y, z)\}$, for each $x, y, z \in X$, is called nonarchimedean, and the space $(X, d)$ is called nonarchimedean quasi-metrizable. As is well known, nonarchimedean quasi-metrizable spaces are characterized as spaces that have a $\sigma$-interior-preserving base. 
COROLlaRY 2.6. Let $X$ be a developable space. Then $X$ is d-IP-expandable if and only if $X$ is nonarchimedean quasi-metrizable. 14].

PrOOF. Both if and only if parts follow easily from Theorem 2.5 and [4, Theorem

It follows directly from the preceding result that every nonarchimedean quasimetrizable space is $\sigma$-orthocompact, but the converse is not true $[4, p .116]$. On the other hand, Sorgenfrey lines show that nonarchimedean quasi-metrizable spaces need not be developable. The following is not known:

QUESTION 2.7. If a space $X$ is developable and quasi-metrizable, then is $X$ $d$-IP-expandable?

This is equivalent to the well-known problem, due to Junnila, whether every developable quasi-metrizable space is nonarchimedean quasi-metrizable.

THEOREM 2.8. For a space $X$, the following are equivalent:

(1) $X$ is an orthocompact developable space.

(2) $X$ has a development $\left\{U_{n}: n \in \mathbf{N}\right\}$ such that each $U_{n}$ is interior-preserving in $X$.

(3) $X$ is a d-IP-expandable developable space.

(4) $X$ is a semistratifiable, nonarchimedean quasi-metrizable space.

PROOF. $(1) \rightarrow(2)$ is trivial. (2) $\rightarrow(3)$ : Under (2), $X$ is a submetacompact $\sigma$ orthocompact space. Then $X$ is orthocompact. By Proposition 2.4, $X$ is $d$-IPexpandable. $(3) \rightarrow(4)$ follows from Corollary 2.6. (4) $\rightarrow(1)$ : Under (4), $X$ is a submetacompact $\sigma$-orthocompact space, and therefore $X$ is orthocompact. Since a semistratifiable $\gamma$-space is developable [7], $X$ is developable.

COROLLARY 2.9. If for each $n \in \mathbf{N}, X_{n}$ is an orthocompact developable space, then so is $\prod_{n=1}^{\infty} X_{n}$.

PROOF. This follows from the fact that semistratifiability and having a $\sigma$ interior-preserving base are countably productive properties.

A space $X$ is said to have property $(\mathrm{P})$ provided that for a closed $G_{\delta}$-set $F$ of $X$, there exists a family $U$ of open subsets of $X$ satisfying the following:

(1) $U /(X-F)$ is interior-preserving in $X-F$.

(2) For each open subset $V$ of $X$, there exists $U \in U$ such that $V \cap F=U \cap F \subset$ $U \subset V$.

THEOREM 2.10. If a space $X$ is nonarchimedean quasi-metrizable, then $X$ has the property $(\mathrm{P})$.

ProOF. Write $F=\bigcap_{n=1}^{\infty} O_{n}$, where for each $n O_{n}$ is open in $X$ and $O_{n+1} \subset O_{n}$. Let $\bigcup_{n=1}^{\infty} B_{n}$ be a base for $X$, where for each $n B_{n} \subset B_{n+1}$ and $B_{n}$ is interiorpreserving in $X$. Let $\{B(\lambda): \lambda \in \Lambda\}$ be the totality of subfamilies of $\bigcup_{n=1}^{\infty}\left(B_{n} / O_{n}\right)$. Then it is easy to see that $U=\{\bigcup B(\lambda): \lambda \in \Lambda\}$ is the desired family.

COROLLARY 2.11. If a space $X$ is perfect and nonarchimedean quasi-metrizable then $X$ is d-IP-expandable.

PROOF. Let $\mathcal{F}=\left\{F_{\lambda}: \lambda \in \Lambda\right\}$ and $\mathcal{U}=\left\{U_{\lambda}: \lambda \in \Lambda\right\}$ be the same pair of families as in Definition 2.1. We apply the theorem to the closed subset $F=\bigcup\left\{F_{\lambda}: \lambda \in \Lambda\right\}$ to get a family $\mathcal{W}$ of open subsets of $X$ satisfying (1) and (2) above with $\mathcal{U}$ replaced by 
W. Observe that for each $F_{\lambda} \cup\left(U_{\lambda}-F\right)=U_{\lambda^{\prime}}$ is open in $X$ such that $F_{\lambda}=U_{\lambda^{\prime}} \cap F$. For each $\lambda$, take $W_{\lambda} \in \mathcal{W}$ such that

$$
W_{\lambda} \cap F=F_{\lambda} \subset W_{\lambda} \subset U_{\lambda^{\prime}} .
$$

Then it is easy to see that $\left\{W_{\lambda}: \lambda \in \Lambda\right\}$ is the IP-expansion of $\mathcal{F}$ with respect to $U$.

We call a family $U$ of open subsets of $X$ an outer base of a subset $F$ in $X$ if for each open subset $O$ with $F \subset O$ there exists $U \in \mathcal{U}$ such that $F \subset U \subset O$.

COROLLARY 2.12. If $X$ is perfect and nonarchimedean quasi-metrizable, then every closed subset $F$ of $X$ has an outer base $\mathcal{U}$ in $X$ such that $\mathcal{U}$ is interiorpreserving in $X-F$.

THEOREM 2.13. Let $X$ be a developable space. Then $X$ is IP-expandable if and only if $X$ is d-IP-expandable.

PROOF. The "only if" part is trivial. "If" part: Let $\mathcal{F}=\left\{F_{\lambda}: \lambda \in \Lambda\right\}$ and $\mathcal{U}=\left\{U_{\lambda}: \lambda \in \Lambda\right\}$ be the same pair of families as in Definition 2.2. Since $X$ is semistatifiable, by the method of [10], we can get a family $\forall=\bigcup_{n=1}^{\infty} \not_{n}$ of closed subsets of $X$ such that each $\mathcal{H}_{n}$ is discrete in $X$ and for each $\lambda$, there exists $\mathcal{H}(\lambda) \subset \mathcal{H}$ such that $F_{\lambda}=\bigcup H(\lambda)$. Write $\mathcal{H}(\lambda)=\bigcup_{n=1}^{\infty} H(\lambda, n)$, where $H(\lambda, n)=H(\lambda) \cap H_{n}$ for each $n$. By Theorem 2.8, $X$ is nonarchimedean quasi-metrizable. Therefore, by Corollary 2.10 , each $H \in \not$ has an outer base $\mathcal{U}(H)$ in $X$ such that $\mathcal{U}(H)$ is interior-preserving in $X-H$. For each $\lambda \in \Lambda$ and each $H \in \mathcal{H}(\lambda, n), n \in \mathbf{N}$, we choose $U(H) \in \mathcal{U}(H)$ such that $U(H) \subset U_{\lambda} \cap O_{n}\left(F_{\lambda}\right) . \quad\left(\left\{O_{n}(F): n \in \mathbf{N}\right\}\right.$ is the semistratification of $F$ in $X$.) Set

$$
W_{\lambda}=\bigcup\{U(H): H \in \mathcal{H}(\lambda)\} .
$$

Then it is easy to see that $F_{\lambda} \subset W_{\lambda} \subset U_{\lambda}$ for each $\lambda$. To see that $\left\{W_{\lambda}-F_{\lambda}: \lambda \in \Lambda\right\}$ is interior-preserving in $X$, let $p \in \bigcap\left\{W_{\lambda}-F_{\lambda}: \lambda \in \Lambda_{0}\right\}$ for $\Lambda_{0} \subset \Lambda$. There exists $n \in \mathbf{N}$ such that $p \in X-O_{n}\left(\bigcup\left\{F_{\lambda}: \lambda \in \Lambda_{0}\right\}\right)$. Since

$$
\bigcup_{k=1}^{n-1}\left[U(H): H \in \bigcup\left\{H(\lambda, k): \lambda \in \Lambda_{0}\right\}\right]
$$

is interior-preserving at $p$, we obtain an open set $O$ of $X$ such that $p \in O \subset$ $\bigcap\left\{W_{\lambda}-F_{\lambda}: \lambda \in \Lambda_{0}\right\}$.

Nagami introduced the class of $L$-spaces, which lies between the classes of Lašnev spaces and $M_{1}$-spaces [6]. He called a space $X$ an $L$-space if $X$ is a paracompact $\sigma$-space such that each closed subset $F$ of $X$ has a closure-preserving outer base and at the same time has an outer base which is interior-preserving in $X-F$. From the definition, we easily have the following result.

THEOREM 2.14. Let $X$ be a stratifiable space. Then $X$ is an L-space if and only if $X$ is IP-expandable.

There exists a stratifiable space $X$ which is not an $L$-space [6, Example 2.2]. Therefore, $d$-IP-expandability need not imply IP-expandability even if $X$ is orthocompact.

Following [2], a space $X$ is called $D$-expandable if for any discrete family $\left\{F_{\lambda}: \lambda \in\right.$ $\Lambda$ \} of closed subsets of $X$ and each family $\left\{U_{\lambda}: \lambda \in \Lambda\right\}$ of open subsets of $X$ such 
that $F_{\lambda} \subset U_{\lambda}$ for each $\lambda$ and $F_{\lambda} \cap U_{\mu}=\varnothing$ whenever $\lambda \neq \mu$, there exists a dissectable family $\mathcal{V}=\left\{V_{\lambda}: \lambda \in \Lambda\right\}$ of open subsets of $X$ such that $F_{\lambda} \subset V_{\lambda} \subset U_{\lambda}$ for each $\lambda$. (For the definition of dissectable families, refer to [2].) Brandenburg showed that a space is $D$-paracompact if and only if it is submetacompact and $D$-expandable [2, Theorem 1].

THEOREM 2.15. If a space $X$ is semistratifiable, then $d$-IP-expandability implies $D$-expandability.

PROOF. It suffices to show that every interior-preserving family $U=\left\{U_{\lambda}: \lambda \in \Lambda\right\}$ of open subsets of a semistratifiable space $X$ is dissectable. Since $\left\{X-U_{\lambda}: \lambda \in \Lambda\right\}$ is a closure-preserving family of closed subsets of $X$, by the method of [10] there exists a family $H=\bigcup_{n=1}^{\infty} H_{n}$ of closed subsets of $X$ satisfying the following:

(1) Each $\mathscr{H}_{n}$ is discrete in $X$.

(2) For each subset $\Lambda_{0} \subset \Lambda$, if $p \in \bigcap\left\{U_{\lambda}: \lambda \in \Lambda_{0}\right\}$ then $p \in H \subset \bigcap\left\{U_{\lambda}: \lambda \in \Lambda_{0}\right\}$ for some $H \in \mathcal{H}$.

For each $n \in \mathbf{N}$ and each $\lambda \in \Lambda$, set

$$
H_{\lambda n}=\bigcup\left\{H \in H_{n}: H \subset U_{\lambda}\right\} .
$$

Then by (2), $U_{\lambda}=\bigcup_{n=1}^{\infty} H_{\lambda n}$ for each $\lambda$. Since $U$ is interior-preserving in $X$, it is easy to see that $\mathcal{U}$ is dissectable in $X$.

However, these notions of expandability are very different, because there exists a nonorthocompact developable space $X$ (for example, $X=\left\langle H_{0}, U\right\rangle$ in [9, Example 4.9]). Therefore, $D$-expandability need not imply $d$-IP-expandability. Also, there exists a perfect subparacompact nonarchimedean quasi-metrizable space [2, Example 1], which is not $D$-paracompact. Therefore, the converse is also not true.

\section{REFERENCES}

1. C. R. Borges, Expansion of closure-preserving collections and metrizability, Math. Japon. 28 (1983), 67-71.

2. H. Brandenburg, On D-paracompact spaces, Topology Appl. 20 (1985), 17-27.

3. R. F. Gittings, Some remarks on weak covering conditions, Canad. J. Math. 26 (1974), 11521156.

4. J. Kofner, On quasi-metrizability, Topology Proc. 5 (1980), 111-138.

5. E. A. Michael, Point-finite and locally finite coverings, Canad. J. Math. 7 (1955), 275-279.

6. K. Nagami, The equality of dimensions, Fund. Math. 106 (1980), 239-246.

7. S. I. Nedev and M. M. Coban, On the theory of o-metrizable spaces. III, Vestnik Moskov. Univ. Ser. I Mat. Mekh. 27 (1972), 10-15.

8. B. M. Scott, Toward a product theory of orthocompactness, Studies in Topology, Academic Press, 1975, pp. 517-537.

9. __ More about orthocompactness, Topology Proc. 5 (1980), 155-184.

10. S. Siwiec and J. Nagata, A note on nets and metrization, Proc. Japan Acad. 44 (1968), 623627.

11. J. M. Worrel, Jr. and H. H. Wicke, Characterizations of developable topological spaces, Canad. J. Math. 17 (1965), 820-830.

Department of Mathematics, Joetsu University of Education, Joetsu, NiIGATA 943, JAPAN 